\title{
The Long-Term Prognosisc of Chronic Kidney Disease in Patients with Atrial Fibrillation Undergoing Coronary Stenting
}

\author{
Yu Bin Wang' ${ }^{1}$ Juan $\mathrm{Ma}^{2}$, Jian Yong Zheng', Bo Yang Zhang ${ }^{3}$, Peng Fei Liu ${ }^{3}$, Nan Nan Wang ${ }^{3}$, Guang Yuan Song1, Jian \\ Min Chu' ${ }^{1}$, Cheng Jun $\mathrm{Guo}^{4}$, Tian Chang $\mathrm{Li}^{3}$ and Yong Jian $\mathrm{Wu}^{* 1}$
}

${ }^{1}$ Department of Cardiology, Fuwai Hospital, People's Republic of China

${ }^{2}$ Weifang medical college Weifang Shandong province of People's Republic of China

${ }^{3}$ Department of Cardiology, PLA Navy General Hospital, People's Republic of China

${ }^{4}$ Department of Cardiology, Capital Medical University, People's Republic of China

Received: August 01, 2018; Published: August 10, 2018

*Corresponding author: Yong Jian Wu, Fuwai Hospital, National Center for Cardiovascular Diseases, Chinese Academy of Medical Sciences, No. 167 Beilishi Road, Xicheng District, Beijing, People's Republic of China 100037, China

Tian-Chang Li, Department of Cardiology, PLA Navy General Hospital, No. 6 Fucheng Road, Haidian District, Beijing, People’s Republic of China. Postal Code: 100048, China

\begin{abstract}
Background: The effect of chronic kidney disease (CKD) on the long-term prognosis of patients with atrial fibrillation (AF) undergoing coronary stenting was less studied.

Methods: We enrolled 2,511 patients with nonvalvular AF undergoing coronary stenting between January 2010 and June 2015 from 12 hospitals in Beijing, China

Results: $22.9 \%$ had CKD (creatinine clearance $<60 \mathrm{ml} / \mathrm{min}$ ). Compared to those with preserved renal function, patients with CKD were older, and had the higher prevalence of women, hypertension, previous ischemic stroke, cardiac dysfunction, and anemia. All patients were treated with drug-eluting stents. Dual antiplatelet therapy was the dominant antithrombotic strategy in both groups $(96.0 \%$ vs. $93.9 \%$, $\mathrm{P}=0.054)$. The follow-up duration was $39.5 \pm 18.6$ months. Complete follow-up data was obtained for $95.3 \%$ of this cohort. CKD group had higher incidences of death $(19.0 \%$ vs. $6.9 \%, \mathrm{P}<0.001$ ), ischemic stroke ( $5.5 \%$ vs. $3.3 \%, \mathrm{P}=0.020$ ), MACCE (a composite of all-cause death, non-fatal myocardial infarction, target vessel revascularization, ischemic stroke and arterial thromboembolism, $28.2 \%$ vs. $14.7 \%, \mathrm{P}<0.001$ ) and Bleeding Academic Research Consortium (BARC) $\geq$ grade $3(2.4 \%$ vs. $0.8 \%, \mathrm{P}=0.003)$. No significant difference was noted with regard to myocardial infarction and target vessel revascularization. Cox multivariate regression identified CKD as an independent risk factor for all-cause death (Hazard ratio [HR]: 1.85, 95\%CI: 1.37-2.50), MACCE (HR: 1.56, 95\%CI: $1.25-1.96$ ) and BARC $\geq 3$ bleeding (HR: 3.14, 95\%CI: 1.49-6.61), but not for ischemic stroke (HR: 1.10, 95\%CI: 0.67-1.79).
\end{abstract}

Conclusion: CKD was independently associated with poor long-term prognosis except for ischemic stroke in patients with AF and coronary stenting.

Keywords: Atrial Fibrillation; Percutaneous Coronary Intervention; Chronic Kidney Disease; Prognosis

Abbreviations: CKD: Chronic Kidney Disease; AF: Atrial Fibrillation; PCI: Percutaneous Coronary Intervention; MACCE: Major Adverse Cardiac/ Cerebrovascular Events; BARC: Bleeding Academic Research Consortium; HR: Hazard Ratio

\section{Introduction}

Approximately $4.5 \%-12.3 \%$ of patients undergoing percutaneous coronary intervention (PCI) have atrial fibrillation (AF) [1-5]. These patients had an increased mortality, thrombotic risk and bleeding complications compared with those without AF, even after adjustment for covariate factors [1-4]. Risk stratification for this specific population could help identify those at increased risk and guide antithrombotic strategies. However, some observation al studies investigating traditional risk scoring systems such as CHA2DS2-VASc and GRACE scores only showed the limited predictive ability for future adverse events [6]. Chronic kidney disease (CKD), usually defined as significantly decreased glomerular filtration rate or creatinine clearance, often accompanies many cardiac diseases and is not an infrequent co-morbidity in patients with $\mathrm{AF}$ and coronary stenting [4]. CKD is often associated with other traditional risk factors for worse cardiovascular outcomes (e.g., diabe- 
tes). However, CKD per se disorders many aspects of the thrombotic process, and complicates the metabolism of many cardiovascular drugs. In this study, we aim to evaluate whether CKD independently affects the clinical outcomes (including death, thromboembolic events and bleeding complications) of patients with AF undergoing coronary stenting.

\section{Methods}

We enrolled all patients with concomitant coronary heart disease and previously documented nonvalvular AF who underwent PCI with stenting between January 2010 and June 2015 in 12 hospitals of Beijing, China. We calculated creatinine clearance for each patient with the Cockcroft-Gault equation. Exclusion criteria were history of intracranial bleeding; cardiogenic shock; peptic ulcer; thrombo cytopenia (platelet concentration lower than $50 \times 10^{9} / \mathrm{L}$ ); major bleeding (according to the Thrombolysis in Myocardial Infarction [TIMI] criteria) in the past 12 months ; cancer in any organ and severe lung/liver disease All eligible patients were divided into two groups according to their renal function:

1) CKD group, with creatinine clearance $<60 \mathrm{ml} / \mathrm{min}$; and

2) non-CKD group, $\geq 60 \mathrm{ml} / \mathrm{min}$.

All patients were followed up in the outpatient departments or by telephone. Each death was confirmed with the National Demographic Registry. We defined the major adverse cardiac/ cerebrovascular events (MACCE) as a composite of all-cause death, non-fatal myocardial infarction, target vessel revascularization, ischemic stroke and other peripheral artery thromboembolism. We graded bleeding events according to Bleeding Academic Research Consortium (BARC) criteria, and regarded grade 2 or higher as a major bleeding event. We also noted occurrence of either MACCE or any major bleeding complication as a combined endpoint. The study was conducted in accordance with the ethical guidelines of the Declaration of Helsinki. The ethics committee of our center approved the study protocol, and all patients gave informed written consent to participate in the study. The statistical analysis was performed with SPSS version 15.0 (Chicago, IL, USA). We used 2 -independent-sample t-test, $\chi 2$ or Fisher exact test for comparison between patients with impaired and preserved renal function. The Hazard ratio (HR) was calculated as a measure of strength for the impact of renal dysfunction on clinical outcomes. Multivariate Cox proportional hazard regression was used to correct for baseline imbalances between the two groups. A two-sided P value of less than 0.05 was considered to be statistically significant.

\section{Results}

A total of 2,511 patients were included in the study with a mean age of $66.6 \pm 9.6$ years and CKD in 576 (22.9\%) patients. The clinical characteristics at baseline according to renal function. Compared to those with preserved renal function, patients with CKD were older, and had the higher prevalence of women, hypertension, previous ischemic stroke and anemia, but were less likely to be current smokers. An insignificantly greater prevalence of acute ST-segment elevation myocardial infarction and multivessel stenting was noted in the CKD group. All patients were treated with drug-eluting stents. Cardiac dysfunction, manifested as symptomatic heart failure or a reduced left ventricular ejection fraction of less than $50 \%$, was also more common in the CKD group. Patients in the CKD group had significantly higher CHA2DS2-VASc, HAS-BLED and GRACE scores than those with preserved renal function. Dual antiplatelet therapy was the dominant antithrombotic strategy in both populations, although CKD group had marginally greater use of dual antiplatelet and less use of triple therapy. Additionally, a small but significant difference also existed with respect to $ß$ receptor blocker, statins and proton-pump inhibitor use. The mean follow-up duration was $39.5 \pm 18.6$ months. Complete follow-up data was obtained for 95.3\% (94.3\% in CKD group and $95.7 \%$ in non-CKD group) of the study population.

A total of 230 (9.6\%) patients died after PCI, and the incidence was $0.6 \%$ for myocardial infarction, $4.7 \%$ for target vessel revascularization, $3.8 \%$ for ischemic stroke and $17.8 \%$ for MACCE. Major bleeding occurred in $72(3.0 \%)$ patients, with an incidence of $1.8 \%$ for BARC grade 2 and $1.2 \%$ for BARC grade 3 or higher. Compared the clinical outcomes after PCI between the two groups. CKD group had a significantly higher incidence of death, ischemic stroke and MACCE. No significant difference was noted with regard to myocardial infarction and target vessel revascularization. A trend towards more major bleeding events was observed in CKD group, and the difference achieved significance at BARC grade 3 or higher. The incidence of the combined endpoint of MACCE and major bleeding was also significantly higher in the CKD group. After multivariate Cox regression, CKD remained an independent risk factor for all-cause death (HR: 1.85, 95\%CI: 1.37-2.50), MACCE (HR: 1.56, 95\%CI: 1.25-1.96), major bleeding (HR: 1.69, 95\%CI: $1.03-$ 2.78), BARC grade 3 or higher bleeding (HR: 3.14, 95\%CI: 1.49-6.61) and the combined endpoint (HR: 1.52, 95\%CI: 1.23-1.88). However, its association with ischemic stroke (HR: 1.07, 95\%CI: 0.65-1.75) was non-significant after correction for baseline imbalances.

\section{Discussion}

This multicenter observational study showed patients with CKD had adverse clinical outcomes compared to those with preserved renal function during a mean follow-up period of 40 months. Multivariate analysis identified CKD as an independent risk factor for all-cause death, MACCE and major bleeding events. In recent years, patients with AF and coronary stenting have appealed to cardiologists due to their worse prognosis and controversy over the optimal antithrombotic strategy. Relative to those without a history of AF, AF patients undergoing coronary stenting often had an advanced age and were more likely to have co-morbidities such as hypertension, diabetes, congestive heart failure or renal insufficiency [2-4]. In our study, the cohort were older (66.6 \pm 9.6 vs. $61.7 \pm 11.4$ years) and had an increased prevalence of hypertension (73.7\% vs. $60.2 \%)$, diabetes (30.5\% vs. $21.8 \%)$ and CKD $(22.9 \%$ vs. $13.1 \%$ ) compared to the study population of a multicenter PCI registry in China [8]. The difference reflected the phenomenon that AF usually occurred with aging and accumulating risk factors. The poor clinical outcomes in this patient population were evident even after adjustment with other traditional risk factors [1-4].

However, few studies have investigated the effects of potential risk factors on the long-term clinical outcomes in this population 
[6]. Renal dysfunction has been recognized as a prognostic factor in acute coronary syndrome irrespective of whether PCI was performed [8-14], and the GRACE score incorporates serum creatinine as a major component for risk stratification. However, its role in assessing the thromboembolic risk for $\mathrm{AF}$ patients has been debated. CHA2DS2-VASc score did not include renal function, while a large-scale study demonstrated that CKD was an independent risk factor for stroke in AF patients with a comparable predictive strength (relative risk: 1.4) to heart failure and advanced age [15]. Similarly, in a Chinese population with nonvalvular AF, CKD was moderately but significantly associated with the risk of stroke or transient ischemic attack after adjustment with the CHADS2 score (odds ratio: 1.005, 95\%CI: 1.002-1.009) [16]. It was still unclear whether the increased thromboembolic risk found in CKD patients resulted from CKD itself or coexistent risk factors closely related to CKD. In this study, patients with CKD were older and had the higher prevalence of hypertension and previous ischemic stroke than those with preserved renal function, constituting a high-risk population for future cerebrovascular events (CHA2DS2-VASc score: $4.2 \pm 1.6$ vs. $2.8 \pm 1.6)$. However, the increased incidence of ischemic stroke in the CKD group (5.2\% relative to $3.2 \%$ for non-CKD) proved to be attributable to other concomitant risk factors. In the AFCAS registry, renal dysfunction was independently associated with 1-year all-cause mortality and MACCE in patients with AF referred for PCI [17].

Our study confirmed this correlation on a long-term followup, and also identified CKD as an independent risk factor for BARC grade 2 or higher bleeding events. In addition to the adverse effect of concomitant risk factors, the worsened cardiovascular outcomes of patients with CKD can be explained with its special pathophysiologic pathways. Disorders of thrombosis could occur in CKD, including excessive thrombin generation and decreased platelet aggregation, therefore increasing both thrombotic and bleeding risks concomitantly [7]. Additionally, pharmacologically induced platelet antagonism can be augmented due to reduced renal excretion of antiplatelet agents. Renal dysfunction promotes inflammation and induces unopposed hyperactivation of neurohormonal signaling pathways (including sympathetic nervous system, rennin-angiotensin-aldosterone system, endothelin and vasopressin). All these pathophysiologic changes worsen ischemia, myocardial dysfunction, and end-organ injury. Dual antiplatelet therapy was used predominantly (94.4\%) in this Chinese population irrespective of estimated thrombotic and bleeding risks. This finding contrasts with previous observational studies from other countries, in which warfarin based therapy (Triple therapy or warfarin with one antiplatelet agent) was the preferred antithrombotic strategy in AF patients undergoing PCI $[18,19]$.

Although there were few large-scale clinical trials to recommend a superior antithrombotic regimen for patients with $\mathrm{AF}$ and coronary stenting, warfarin has been demonstrated to be an effective agent to reduce cerebral thromboembolic risk in this population [20-22]. Actually, warfarin was underused in the general population with AF in China. In a multicenter registry from
50 hospitals in China, $86.2 \%$ of patients with nonvalvular AF had CHADS2 score $\geq 1$, but only $42.6 \%$ were on warfarin [16]. The most common reasons were patient unwillingness to receive regular INR monitoring (43.0\%) and high risk of bleeding (33.3\%) [16]. Another possible reason for the inadequate anticoagulation in this study population was the concern of many Chinese cardiologists of in-stent thrombosis and excessive bleeding after coronary stenting when warfarin was used with antiplatelet agents. There are some limitations to this study. This study was not prospectively designed to assess the long-term prognosis of patients with AF and coronary stenting, and the data utilized for analysis was derived from 12 hospitals in Beijing. As all studies involving multicenter databases and registries, there was no audit of data quality and precision. As an inherent nature of retrospective studies, some demographic and clinical information may be missing, such as body mass index. Not all patients had their post-procedure serum creatinine recorded, and therefore we were unable to determine the prevalence of acute kidney injury and evaluate its effect on clinical outcomes. For patients suspected of having died because of loss of contact for a period, we looked up the national demographic registry to confirm whether they were dead or alive. The cause of death was missing, unclear or inaccurate for a considerable amount of patients in the registry, and therefore we could not discriminate cardiac from noncardiac death. All patients were treated with drug-eluting stents, but the stents came from many different manufacturers and were coated with different drugs, which might exert an influence on the clinical outcomes. The choice of stents and antithrombotic regimens was totally at the treating physician's discretion. However, this 'real-world' nature is the strength of our study.

\section{Conclusion}

CKD represented 1/5 - 1/4 of patients with a history of AF undergoing coronary stenting, and independently predicted allcause death, MACCE and bleeding complication on a long-term follow-up. Although patients with CKD had a higher incidence of ischemic stroke compared to those with preserved renal function, this association became insignificant after adjustment with other covariate factors.

\section{Acknowledgment}

This study was granted and supported by Beijing Municipal Science and Technology Commission [Grant number: Z151100004015205]. The authors sincerely thank Hai-Feng Yuan M.D., Xiu Feng Xie M.D. and Feng Wang M.D. of PLA Navy General Hospital, Dang-Sheng Huang M.D. of PLA 304 Hospital, Yun Tian Li M.D. of PLA 305 Hospital, Shou Li Wang M.D. of PLA 306 Hospital, Yan-Yan Chu M.D. of Beijing Xuanwu Hospital, Shi Guo M.D. of Beijing Dongzhimen Hospital and Yu-Ling Niu M.D. of Fangshan First Hospital of Beijing for participating in and supporting this study.

\section{References}

1. Miura T, Miyashita Y, Motoki H, Hideki Kobayashi, Masanori Kobayashi, et al. (2017) Comparison of mid-term outcomes between patients with and without atrial fibrillation undergoing coronary stenting in the second-generation drug-eluting stent era: from the SHINANO registry. Cardiovasc Interv Ther 32(3): 206-215. 
2. Sutton NR, Seth M, Ruwende C, Gurm HS (2016) Outcomes of Patients With Atrial Fibrillation Undergoing Percutaneous Coronary Intervention. J Am Coll Cardiol 68(9): 895-904.

3. Rohla M, Vennekate CK, Tentzeris I, Freynhofer MK, Farhan S, et al. (2015) Long-term mortality of patients with atrial fibrillation undergoing percutaneous coronary intervention with stent implantation for acute and stable coronary artery disease. Int J Cardiol 184: 108-114.

4. Bramalea P, Cuneo A, Zeymer U, Matthias Hochadel, Gert Richardt, et al. (2013) Prognosis of patients with atrial fibrillation undergoing percutaneous coronary intervention receiving drug eluting stents. Clin Res Cardiol. 102(4): 289-297.

5. Gao F, Zhou YJ, Wang ZJ, Shen H, Liu XL, et al. (2010) Comparison of different antithrombotic regimens for patients with atrial fibrillation undergoing drug-eluting stent implantation. Circ J 74(4): 701-708.

6. Fauchier L, Lecoq C, Ancedy Y, Stamboul K, Saint Etienne C, et al. (2016) Evaluation of 5 Prognostic Scores for Prediction of Stroke, Thromboembolic and Coronary Events, All-Cause Mortality, and Major Adverse Cardiac Events in Patients With Atrial Fibrillation and Coronary Stenting. Am J Cardiol 118(5): 700-707.

7. Hiramoto JS, Katz R, Peralta CA, Ix JH, Fried L, et al. (2012) Inflammation and coagulation markers and kidney function decline: the Multi-Ethnic Study of Atherosclerosis (MESA). Am J Kidney Dis 60(2): 225-232.

8. Huo Y, Ho W (2007) Renal insufficiency and clinical outcomes in patients with acute coronary syndrome undergoing percutaneous coronary intervention: a multi-centre study. Journal of Peking University Health sciences. 39(6): 624-629.

9. Loncar G, Barthelemy O, Berman E, Mathieu Kerneis, Thibault Petroni, et al. (2015) Impact of renal failure on all-cause mortality and other outcomes in patients treated by percutaneous coronary intervention. Arch Cardiovasc Dis 108(11): 554-562.

10. Gupta T, Paul N, Kolte D, Harikrishnan P, Khera S, et al. (2015) Association of chronic renal insufficiency with in-hospital outcomes after percutaneous coronary intervention. J Am Heart Assoc 4(6): e002069.

11. Sabroe JE, Thayssen P, Antonsen L, Mikkel Hougaard, Knud Nørregaard Hansen, et al. (2014) Impact of renal insufficiency on mortality in patients with ST-segment elevation myocardial infarction treated with primary percutaneous coronary intervention. BMC Cardiovasc Disord 14: 15.

12. Parikh PB, Jeremias A, Naidu SS, Sorin J Brener, Fabio Lima BS, et al. (2012) Impact of severity of renal dysfunction on determinants of inhospital mortality among patients undergoing percutaneous coronary intervention. Catheter Cardiovasc Interv 80(3): 352-357.
13. Zhang RY, Zhu ZB, Zhang Q, Zhen Kun Yang, Jian Hu, et al. (2009) Impact of moderate or severe renal insufficiency on long-term outcomes in patients undergoing drug-eluting stent based coronary intervention. Int J Cardiol 136(1): 72-79.

14. Appleby CE, Ivanov J, Lavi S, Mackie K, Horlick EM, et al. (2009) The adverse long-term impact of renal impairment in patients undergoing percutaneous coronary intervention in the drug-eluting stent era. Circ Cardiovasc Interv 2(4): 309-316.

15. Go AS, Fang MC, Udaltsova N, Chang Y, Pomernacki NK, et al. (2009) Impact of proteinuria and glomerular filtration rate on risk of thromboembolism in atrial fibrillation: the anticoagulation and risk factors in atrial fibrillation (ATRIA) study. Circulation 119(10):13631369.

16. Sun Y, Wang Y, Jiang J, Lina Wang, Dayi Hu, et al. (2015) Renal Dysfunction, CHADS2 Score, and Adherence to the Anticoagulant Treatment in Nonvalvular Atrial Fibrillation: A Cross-Sectional Study in Mainland China. Clin Appl Thromb Hemost 23(3).

17. Lahtela HM, Kiviniemi TO, Puurunen MK, Axel Schlitt, Andrea Rubboli, et al. (2015) Renal Impairment and Prognosis of Patients with Atrial Fibrillation Undergoing Coronary Intervention - The AFCAS Trial. PLoS One 10(6): e0128492.

18. Rubboli A, Schlitt A, Kiviniemi T, Fausto Biancari , Pasi P Karjalainen et al. (2014) One-year outcome of patients with atrial fibrillation undergoing coronary artery stenting: an analysis of the AFCAS registry. Clin Cardiol 37(6): 357-364.

19. Ruiz Nodar JM, Marin F, Hurtado JA, Valencia J, Pinar E, et al. (2008) Anticoagulant and antiplatelet therapy use in 426 patients with atrial fibrillation undergoing percutaneous coronary intervention and stent implantation implications for bleeding risk and prognosis. J Am Coll Cardiol 51(8): 818-825.

20. Suh SY, Kang WC, Oh PC, Choi H, Moon CI, et al. (2014) Efficacy and safety of aspirin, clopidogrel, and warfarin after coronary artery stenting in Korean patients with atrial fibrillation. Heart Vessels 29(5): 578-583.

21. Lamberts M, Gislason GH, Olesen JB, Kristensen SL, Schjerning Olsen AM, et al. (2013) Oral Anticoagulation and Antiplatelets in Atrial Fibrillation Patients After Myocardial Infarction and Coronary Intervention. Journal of the American College of Cardiology 62(11): 981-989.

22. Dewilde WJM, Oirbans T, Verheugt FWA, Kelder JC, De Smet BJ, et al. (2013) Use of clopidogrel with or without aspirin in patients taking oral anticoagulant therapy and undergoing percutaneous coronary intervention: an open-label, randomised, controlled trial. The Lancet 381(9872): 1107-1115.
ISSN: 2574-1241

DOI: 10.26717/BJSTR.2018.07.001560

Yong Jian Wu. Biomed J Sci \& Tech Res

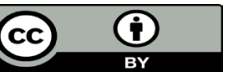

This work is licensed under Creative Commons Attribution 4.0 License

Submission Link: https://biomedres.us/submit-manuscript.php

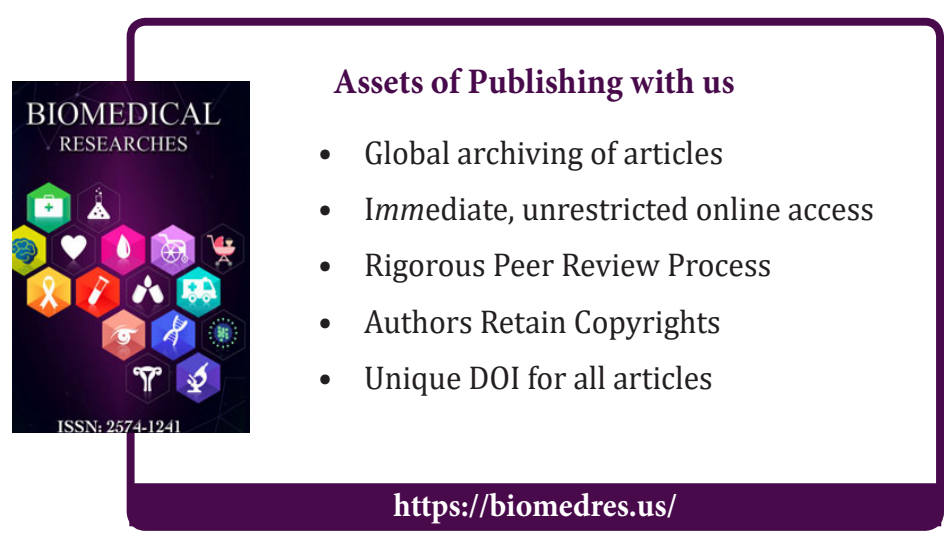

Cite this article: Yu Bin W, Juan M, Jian Y Z, Bo Yang Z, Peng FL, Nan N W, Guang Y S, Jian MC, Cheng J G, Tian CL,Yong J W. The Long-Term Prognosisc of Chronic Kidney Disease in Patients with Atrial Fibrillation Undergoing Coronary Stenting. Biomed J Sci\&Tech Res 7(5)- 2018. BJSTR MS.ID.001560. DOI: 10.26717/ BJSTR.2018.07.001560. 